\title{
Ansiedad de Separación: Tratamiento por Inhibición Recíproca del Rechazo Escolar - Descripción de una Experiencia
}

\author{
Dr. Ricardo Fuentealba H, 1-2
}

\section{Reciprocal Inhibition in the Treatment of School Refusal}

\begin{abstract}
The current concepts about school refusal are revirwed and the therapeutic applications of "Rociprocal Inhibition" as a variant of "Systematic Desensitization" in the treutment of this problem is presented. In a six years old girl in which other therapeutic methods has failed, a successtul axtempt was done with "Reciprocat Inhibition". The first step consisted in the identification of ten alxiety provoking situations related to shool attendance. Then scveral sessions were carried oul at home at the time of departure to school, advancing step by step through out the conflicting stibes, stopping when anxiety symptoms became severe till the next school day. At the end of ten sessions the patient was able to stay at school without the therapist's intervention and fiee of anxiety. Two yejrs later she is a high achievement, normally attending school girl that firecuently participates in extracuricular activities. Thus "Reciprocal Inhibition" techniques seem uscful in some cases of school refusal.

(Key words: School refusial. Reciprocal inhibition therapy).
\end{abstract}

Broadwin, en 1932, describió por primera vez lo que consideró una variante de vagabundeo, que fue llamada Fobia Escolar, por algunos y Rechazo Escolar, por otros. Su descripción original no ha sido básicamente modificada por trabajos posteriores:

"El niño está ausente de la escuela por periodos que varían desde varios meses a un año. La ausencia es consistente: En todo momento los padres saben donde está. Eso es con la madre o cerca del hogar. La razón para el vagabundeo es incomprensible para los padres y la escuela. El niño puede decir que él teme ir a la escuela, teme al profesor, o decir que no sabe por qué no va a la escuela. En el hogar es feliz y aparentemente libre de temores. Cuando es llevado a la escuela se siente miserable, temeroso y a la primera oportunidad corre al hogar a pesar de la certeza de un castigo corporal. La aparición es generalmente súbita. El trabajo escolar y la conducta previa han sido buenos"

En muchos trabajos se ha repetido la observación inicial del mišmo autor, de que estos ninos temen que alguna cosa terrible le ocurra a su madre, lo que los hace correr al hogar para asegurarse de que no es asi y aliviar la ansiedad.

De modo que el aparente ternor a la escuela, sería más bien un temor a dejar la casa.

1 Médico Becado de Neuropsiyuiatría Infantil. Servicio de Salud Mental Infantil Hospital Exequiel González Cortés.

2 Presentado en el II Congreso de Psiquiatría y Neurologia de la Infancia y Adolescencia. Santiago, Septiembre de 1984.
Hay evidencias de que los niños con este problema dejados sin tratamiento, tendrían una mayor frecuencia de estados fóbicos y neurosis en la edad adulta2.3.

EI Rechazo Escolar es un síndrome que no tiene una etiologia, psicopatología, curso, pronóstico y tratamiento uniforme; sino que está asociado a variados trastornos psiquiátricos.

El comienzo generalmente ocurre con quejas vagas o molestias somáticas en el momento de ir a la escuela ${ }^{4}$, que progresan hasta la negativa a ir 0 a permanecer en ella acompanada de ansjedad $y$ aún pánico. Pueden haber factores precipitantes $^{5}$. Se desconoce la prevalencia en nuestro medio. En un estudio de la Isla de Wight ocurrió en $23 \%$ de los niños con trastornos psiquátri$\cos ^{6}$. En los mecanismos psicológicos, es central la ansiedad que se desarrolla a partir de una relación madre-hijo patológica, de dependencia hostil mutua, con una intensa necesidad, por ambos, de proximidad fisica. El término "ansiedad de separación": fue usado para describir la respuesta a los intentos de alterar este estado emocional patológico con presiones para ir a la escuela. También se ha propuesto el término "trastorno por angustia de separación" ". Ia de. presión puede estar presente en estos niños y debe ser buscada activamente ${ }^{8}$.

\section{Interaccion Familiar:}

En la mayoría de los casos de rechazo escolar, estarian presentes uno o más de cuatro princjpales patrones de interacción familiar:

En el primero la madre o más raramente el padre 
sufre de ansiedad crónica en su relación con figuras cercanas, y retiene al niño en casa para su compañía.

En otros casos, el niño teme que algo terrible le suceda a su madre o a su padre mientras èl está en la escuela $y$ permanece en el hogar para prevenir aquello.

Otras veces el pequeño teme que algo terrible le suceda a él si se aleja de la casa y por tanto permanece en ésta.

Finalmente, puede ocurrir que la madre o más raramente el padre temen que algo terrible le suceda al niño mientras está en la escuela, y lo mantienen en la casa.

Parece ser frecuente la ansiedad materna en un ambiente de disarmonía marital e inconsistencia parental. Estos nif̄os tendrían menos experiencias de ausencia materna y son más pasivos, dependientes y sobreprotegidos que sus pares ${ }^{9}$.

\section{Teoria del aprendizaje y terapia conductual de la ansieded de separación:}

Mary Cover Jones en 1924 usó exitosamente la respuesta "alimentación" en un procedimiento de desensibilización. Posteriormente Wolpe (1954, 1958, 1966) en su clásico procedimiento de desensibilización, utilizó la relajación muscular, según la técnica descrita por Jacobson (1938) y la hipnosis como estados inhibidores de la ansiedad ${ }^{10}$.

Lazarus en 1960 describió el uso de la desensibilización sistemática, utilizando una jerarquía centrada en el tema de la separación de la madre. Luego se ha usado la variante llamada "imagineria emotiva", en la que la imagen de un héroe brinda ayuda en las situaciones de ansiedad 11 .

A estas técnicas han seguido la desensibilización in vivo, la terapia implosiva, métodos de condicionamiento por aproximaciones sucesivas y la inhibición reciproca.

\section{Inhibición reciproca:}

Esta técnica es una modificación del procedimiento de desensibilización de Wolpe, en por to menos tres aspectos básicos:

No se usa hipnosis; en lugar del método de relajación, que puede ser difícil de emplear en niños pequeños que no cooperan, se utiliza la respuesta "alimentación" como estado físiológico antagónico a la ansiedad; no se le pide al paciente que se imagine las condiciones que le producen ansiedad, sino que es expuesto gradualmente a una serie de situaciones que involucran el estímulo problema ${ }^{1-12}$.

\section{Caso Clínico}

La paciente es una niña de 6 años de edad, traj́da por su madre al Consultorio Externo de Salud Mental Infantil, porque se resiste a asistir a la escuela. Cursó sin dificultades el Kindergarten el año anterior, en la misma escuela. Ingresó a 10 básico en Marzo de 1982, asistiendo durante 2 meses a clases sir molestias aparentes. En Mayo comenzó a presentar resistencia progresiva a quedarse en la escuela, Ilorando y mostrándose muy ansiosa, por lo que la madre la acompañaba, quedándose en el pasillo contiguo a la sala de clases. Su resistencia aumentó hasta negirse a asistir e incluso vestir el uniforme escolar. Si era forzada a asistir al establecimiento, corría fuera de éste a la menor oportunidad. Faltó a clases durante un mes, reintegrándose acompañadia de su hermana mayor (10 años), qujen se quedaba con ella en la sala de clases tuda la jornada.

Tenía además temor de que su hermana y su madre "se convirtieran en monstruos", mientras ella estaba en la escuela, no permitía a su madre salir de la casa sin ella y se quejaba de dolor abdominal y náuseas a la hora de ir a clases. Frecuentemente tenia pesadillas en las que sus parientes sufrian la temida metamorfosis, mientras ella asistía a clases.

El Rendimiento escolar ha sido en todo momento bueno, preocupándose incluso durante el mes de inasistencia de las tareas y materias. No se encontraron hechos desencadenantes claros. Poco después del inicio de los síntomas la profesora del curso había sido cambiada.

El grupo familiar de la paciente estaba com. puesto por su hermana mayor, su madre 28 años, separada desde hace 3 años) y los abuelos ntaternos. La niña dormía con su hermana. Nunca habia alojado fuera del hogar. No aceptaba quedarse sola en casa, aún por períodos muy breves de tiempo.

No se registraban antecedentes familiares de un problema semejante.

Se habian realizado intentos terapéuticos anteriores los que se basaron en cambiar los eventos reforzantes de la conducta, tales como actitud parental cloando ta niña no asistía a clases, y los juegos, televisión, golosinas, etc., disponibles para ella en esas horas. Al mismo tiempo se debían aumentar los refuerzos en relación con su ida a clases. Además se buscó incentivar sus actividades con riňas de su edad del vecindario, que le permitieran una mayor independencia de su grupo familiar. También se la incluyó en sesiones de terapia ocupacional, en un grupo de nifíos de su edad con problemas de tipo emocional o de desarrollo de habilidades sociales.

Cinco semanas después no habian cambios evidentes en la actitud de la niña hacia la escuela; 
huía de la sala de terapia ocupacional si su madre no entraba con ella y no se logró su participación en las actividades del grupo.

Se hicieron nuevas indicaciones consistentes en que la hermana que la acompañaba en la clase, se iba a retirar 15 minutos antes del término de la jornada, explicándole a la paciente que la esperaría en el pasillo contiguo; dicho periodo de tiempo se aumentaría en 10 minutos cada dos dias.

Luego de dos semanas también se consideró fracasado este intento. La niña oponía tenaz resistencia a que su hermana saliera de la sala, interrumpiendo la clase con su berrinche; tinalmente se negó a levantarse en las maxinas $y$ asistir a la escuela, a menos que le prometieran que su hermana no saldria de la sala.

Se decidió entonces utilizar la técnica de Inhibición Reciproca, ya que la ansiedad es el sintoma principal en la paciente y se presenta en una actividad susceptíble de ser organizada en una jerarquia de situaciones ansiógenas.

De acuerdo con la niña y su madrc, se eligió el chocolate como alimento para ser consunido en las situaciones de ansiedad. Como signos de ansiedad se consideraron la sudoración de las manos, inquiet ud motora, silencio inesperado, negarse a caminar, o la expresión verbal de temor o deseos de volver a casa.

Se informó a las autoridades del establecimiento $y$ a la profesora del procedimiento que se seguiría, con el propósito de obtener su colaboración.

Con la participación de la paciente y su madre se definieron las situaciones de ansiedad en la actividad problema que se ilustra en la Tabla 1 .

Tabla 1.

Situacionts de ansiedad ante la asistencia a la escuela

1. Levantarse vistiendo el uniforme escolar;

2. Salir de la casa:

3. Comenzar a caminar en dirección a la escuela;

4. Llegar a una cuadra de distancia de su casi, Jugar donde perdía de vista a esta;

5. Llegar a una cuadra de la escuela, sitio donde comenzaba a verla;

6. Llegar a más o menos 10 métros de la puerta del establecimiento;

7. Pasar la puerta de éste;

8. Caminar hacia la sala de clases;

9. Entrar en la sala;

10. Permanecer èn su interior.

Las sesiones se realizaron diariamente, partiendo desde el domicilio de la paciente a las 08:15 hora en que habitualmente iba a clases. En cada sesión se avanzó hasta que la niña mostrara signos intensos de ansiedad, que requirieran de algunos minutos para que se tranquilizara. Si a juicio del autor la ansiedad era leve y cedia rápidamente con la ingestión de chocolate, se continuaba hasta la situación siguiente.

Sesion 1: Aceptó vestir el uniforme escolar sin problemas, al parecer porque su hermana hizo lo mismo junto a ella. Cuando yo llego, se niega a salir de la casa, con intensas muestras de ansiedad; le doy chocolate y espero que se tranquilice.

Sesión 2: La madre le dio chocolate en la situación 1 . Se le asegura que hoy no irá a clases y no llevará su bolso escolar.

Avanzamos hasta la situación 4, necesitando chocolate en los números 2,3 y 4 .

Sesion 3: Hoy llegamos hasta la situación 5; se le dio chocolate en lugar 4 , en un punto intermedio entre ésta y el número $5, y$ en este último lugar. No mostró ansiedad en las tres primeras situaciones. No llevó su bolso.

Sesion 4: Accedió a llevar su bolso escolar. Avanzamos hasta el numero 8 ; se mostró ansiosa sólo en las situaciones 7 y 8 . No pareció afectada por la situación 6 .

Sesión 5: Hoy la escuela no tuvo clases. Avanzamos hasta la situación 10. Fue necesario darle chocolate en los sitios 7 y 9; además una dosis extra al encontrarnos con su profesora.

Sesion 6: Igual a la anterior en un día de clases normal. Permanecimos la primera hora en clases, en pupitres separados. Se mostró ansiosa en las situaciones $8,9,10$ y cuando su maestra le revisa las tareas.

Sesión 7: Similar a la anterior, excepto que salí de la sala 20 minutos antes que ella, esperándola en el pasillo. Se le dio chocolate en los lugares 8,9 y 10 .

Sesión 8: Esta vez fue llevada por su madre durante todo el trayecto.

Se necesitó chocolate y la intervención del terapeuta (que las seguía a cierta distancia) en la situación 10 . $\mathrm{Se}$ quedó sola en clases toda la mañana. 
Sesión 9: Fue similar a la anterior. La madre le dio chocolate en la situación 10. EI terapeuta no intervino.

Sesión 10: La madre la llevó a clases sin que la paciente mostrara ansiedad en ningún momento. El terapeuta no intervino.

Dos y cuatro semanas después fue observada desde cierta distancia su asistencia a clases, sin aviso previo a ella ni a su familia. Parecía tranquila $y$ contenta, lo que fue posteriormente confirmado por su madre, además de informar de que mantenía un buen rendimiento escolar.

Un año y dos meses después se la visjtó en su casa (noviembre de 1983). Cursaba $2^{\circ}$ básico con excelentes calificaciones, no habia vuelto a mostrac rechazo por la escuela y tenia buenas relaciones con sus pares. Continuaba con la misma profesora.

Se efectuó otra visita dos años después del tratamiento (septiembre de 1984); asistía sin problemas a $3^{\circ}$ básico, manteniendo buenas caljficaciones; participaba en un grupo de catecisino fuera del horario de clases. Seguía con la misma profesora, a quién ayudaba en tareas menores durante la jornada escolar.

Se la invitó a incorporarse a un grupo de niños en sesiones de Terapia Ocupacional, con la intención de comparar su conducta actual con la observada hace dos años. Aceptó gustosa y desde la primera sesión entabló amena charla con sus pares, tomando la iniciativa en los juegos y no dio muestras de ansiedad.

"Cuando grande" quiere ser... profesora.

\section{DISCUSION}

De acuerdo con la literatura, los tesultados de distintos tratamientos del rechazo escolar son buenos. E1 pronóstico parece mís bien estar en relación a la severidad del trastorno, la edad del niño $y$ el tiempo transcurrido entre la aparición de sintomas y el comienzo del tratamiento ${ }^{1}$.

Es importante la evaluación acuciosa del paciente, su nivel intelectual, rendimiento escolar, dificultades especificas, interacción familiar, características del establecimiento educacional, etc.

En el manejo mismo parece esencial lograr una relación de confianza con el niño, y obtener la participación activa de su grupo familiar y de las autoridades escolares, insistiendo en un pronto retorno a la escuela (Yates).

No parece posible que el procedimiento descrito pueda ser realizado por un familiar cercano, lo que implica la necesidad de que el terapeuta trabaje en terreno y pudiera ser ésta la principal objeción a este método.

En la experiencia presentada, el cambio de la situación problema, se obtuvo rápidamente $y$ ha perdurado hasta hoy; además se ha generalizado a otras actividades de la niña, quien ha logrado una mayor independencia de su madre y ha cambiado su actitud frente a sus pares.

Aún cuando en estos resultados puedan estar influyendo factores como el tiempo y otros que no hayan sido medidos, me atrevo a recomendar el método terapéutico cuando lo permitan las condiciones del paciente y del terapeuta interesado.

\section{RESUMEN}

Se presenta una revisión de los conceptos actuales de rechazo escolar y sobre la técnica de la inhibición recíproca, como variante de la desensibilización sistemática descrita por Wolpe.

Se describe posteriormente un caso de Rechazo Escolar y los intentos terapéuticos previos, el método actual, sus resultados y el seguimiento de dos años.

Se concluye que la têcnica es recomendable en ciertos casos de recilazo escolar.

\section{REFERENCIAS}

'Broudivin I.: A contribution to the study of truancy. Amer. J. Orthopsychiat. 2: 253, 1932.

2 Johnson A., Falstein E., Szureck S., Svensen M.: "School Phobia". Amer. J. Orthopsychiat. 11: 702, 1941 .

${ }^{3}$ Warren $W$ : Some retationships between the psychiatry of children and of adults. The $J$. of Mental Science 106: $815,1960$.

4 Bain H.: Chronic Vague Abdominal Pain in Children. Pediatr. Clin. North Am. 21: 995, 1974.

5 Parquet P.H., Delcambre G.: Les lhobies Scolatres. Lille Med. 25: 586, 1980.

- Rutter M.: Child Psychiatry. London, Blackwell Scientific Publications, 1977. pag. 455-86.

? American Psychiatric Assaciation.: Diagnostic and Statistical Manual of Mental Disorders. 3rd Ed. Washington D.C. APA, 1980. pág. 228-30.

${ }^{8}$ Ruberstein J., Hastings E.M.: School refusal in adolercence; undestanding the symptom. Adolescence 15: 775, 1980.

${ }^{9}$ Berg 1., Butler A., Fairbairn I., Mc. Guire R.: The parents of school phobic adolescents a preliminary investigation of family iffe variables. Psychol. Med. 11: 79,1981

1 I Yates $A_{\text {.: }}$ Terapia del comportamiento. 4a. Reimpr. Fditorial Triltas, México, 1978. pág. 175-86.

11 Montenegro H.: Severe separation insiety in two preschool children; successfully treated by reciprocal inhibition. J. Child. Psychol. Psychiat. 9: 93, 1968.

12 tyallace $W$ : Desensibilization; social and cognitive factors underlying the effectivene'ss of Wolpan's Procedure. Psychol Bulletin 76: 311, 1971. 